\title{
Gestão do conhecimento em organizações públicas de saúde no Brasil: diagnóstico de práticas
}

G erson Rosenberg, Pierre 0 hayon e Fábio Ferreira Batista

\section{Introdução}

Mudanças importantes aconteceram no final do século XX resultantes do processo de globalização. Uma dessas se refere à maneira como o conhecimento técnico e científico vem sendo usado pelas instituições com relação aos seguintes aspectos: identificação, compartilhamento, difusão, controle, manutenção, apropriação e seu desenvolvimento (Rod Rig ues apud Fleury e O Liveira JR., 2001).

Em 2002 foi realizado um estudo em algumas organizações públicas dos países que compõem a Organização para a Cooperação e Desenvolvimento Econômico $(\mathrm{O} \mathrm{CDE})$ com 0 intuito de obter informações comparativas em nível nacional e setorial sobre as práticas de G estão do Conhecimento (GC) utilizadas. Este concluiu que as mudanças organizacionais do setor público estão ocorrendo em decorrência das práticas de GC adotadas. Há uma percepção que a maior eficiência das organizações que recorrem a essas práticas é devida principalmente ao maior grau de relacionamento entre as instituições, ao aumento da 
transparência dos processos institucionais e ao crescimento dos investimentos nas tecnologias de informação e comunicação (OCDE, 2002).

No Brasil, também foram realizados, a partir de 2004, três estudos sobre GC pelo Instituto de Pesquisa Econômica Aplicada (IPeA). 0 primeiro foi 0 $\mathrm{G}$ overno que $\mathrm{A}$ prende: $\mathrm{G}$ estão do $\mathrm{C}$ onhecimento em 0 rganizações do E x ecutivo Federal, no qual se discutiu a importância do conceito da Gestão do Conhecimento (GC) para a administração pública e identificou-se o estágio de sua implementação em seis organizações públicas: Banco do Brasil, Caixa Econômica Federal, Serviço Federal de Processamento de Dados (Serpro), Petróleo Brasileiro S.A. (Petrobras), Empresa Brasileira de Pesquisa Agropecuária (E mbrapa) e Banco Central do Brasil. Em sua conclusão, foram listados alguns fatores críticos para a institucionalização da G C em órgãos e entidades da Administração Pública (BATISTA, 2004).

$\mathrm{O}$ segundo trabalho, intitulado $\mathrm{G}$ estão do Conhecimento na A dministração Pública, analisou as mudanças que estão ocorrendo na gestão pública quanto à implementação de práticas de GC em vinte e oito órgãos da administração direta e em seis empresas estatais do Executivo federal brasileiro. Este possibilitou uma avaliação da situação atual das práticas de $\mathrm{GC}$ na administração pública direta do Governo Federal,abordou estratégias de implantação da GC, comparou a situação do governo brasileiro com os governos dos países membros da OCDE participantes de pesquisa realizada em 2002 e propôs, ainda, recomendações e diretrizes para a política de governo eletrônico em GC (BATisTa et al., 2005).

Finalmente, o terceiro trabalho foi 0 D esafio da $\mathrm{G}$ estão do $\mathrm{C}$ onhecimento nas Á reas de A dministração ePlanejamento das Instituicões Federais de E nsino Superior (IFE S) e teve como finalidade avaliar como as áreas de administração e planejamento de quarenta e cinco Instituições Federais de Ensino Superior (Ifes) tratam o tema da GC (BATISTa, 2006), permitindo verificar 0 estágio em que se encontravam as IFES quanto à formalização e explicitação da GC e propor recomendações sobre as ações que seus dirigentes deveriam adotar para a consolidação do processo de GC.

D essa forma, um estudo sobre GC no setor público brasileiro na área da saúde se torna importante não só pela possibilidade da comparação com os outros estudos já realizados, mas também como uma análise que possa ajudar as organizações de saúde a melhorarem sua gestão. O s objetivos do estudo foram: (i) identificar as práticas de GC tomando como base os requisitos item 4.3 do critério 4 do Modelo de Excelência em G estão Pública; (ii) verificar o grau de adequação e disseminação de GC usadas pelos Centros de Pesquisas Clínicas e pelas organizações de saúde adesas ao Gespública (Gestão Pública e D esburocratização); e (iii) sugerir recomendações para a divulgação do Modelo de Excelência em G estão Pública na área da saúde.

\section{Aspectos conceituais do estudo}

0 conhecimento existente dentro das organizações é seu ativo mais importante, quer seja individual ou coletivo, ainda que não contabilizado no sistema de informação econômico-financeiro tradicional. Assim, o conhecimento não aparece em balanços patrimoniais, apesar de estar diretamente ligado aos indicadores de mercado, crescimento e rentabilidade (TERRA, 2001, p. 54). A GC 
nas organizações passa pela compreensão das características e demandas do ambiente competitivo, onde cada vez mais o clima turbulento faz com que as vantagens competitivas precisem ser constantemente reinventadas. Ela está intrinsecamente ligada à capacidade das organizações em utilizar e combinar as várias fontes e tipos de conhecimento organizacional (NoNAKA e TAKEUCHI, 1997).

A G estão do Conhecimento se interliga com outras áreas, notavelmente a gestão da informação e de pessoas, com os níveis de percepção que cada uma delas pode oferecer. Em ambas as áreas, os pontos em comum são os dos fluxos de informação e de análise apoiados em tecnologias e pessoas (SveiBy, 2001). Podese então afirmar que GC possui caráter universal, pois se aplica a qualquer organização pública ou privada. Como resposta ao modo de gerenciar o conhecimento dentro das organizações é que surgiu a Gestão do Conhecimento, abordada sob diferentes focos na literatura organizacional, como segue: (i) aprendizado individual e organizacional (cultura organizacional); (ii) relações entre pessoas; (iii) desenvolvimento de competências individuais; (iv) mapeamento, codificação e compartilhamento do conhecimento organizacional; (v) conectividade entre as pessoas; (vi) alavancagem dos avanços em informática e em telecomunicações; (vii) mensuração do capital intelectual; e (viii) conhecimento coletivo e individual, implícito ou explícito, estoque ou fluxo e, finalmente, interno ou externo (Terra apud Fleury e O liveira Jr., 2001).

Terra (2001, p. 82) considera a GC como a garantia de que todos tenham acesso ao conhecimento da instituição, quando, onde e na forma de que necessitam. D avenport et al. (2004, p. 65) consideram a GC como "processos, métodos e sistemas para gerar, coletar, analisar, organizar, difundir e aplicar informações, entendimentos e experiências coletivas passadas, presentes e, às vezes, futuras, em benefício de uma empresa ou sociedade" . A aceleração dos processos de geração e codificação de conhecimentos está associada à difusão das tecnologias da informação e comunicação (TICs), que possibilitam alavancar um conjunto de

"Os serviços públicos de saúde fundamentados no modelo biomédico e hospitalocêntrico estão sendo duramente questionados na maneira como vêm funcionando atualmente. Percebe-se que este modelo com uma visão especializada e focal do processo saúde-doença está ultrapassado."

inovações de produto, processos organizacionais, sociais e institucionais nos diversos setores da economia. 0 novo modo de gerenciar fundamentado na GC não se restringe aos setores de ponta, mas possui um caráter bastante amplo que muda o modo como o ser humano aprende, produz, trabalha, consome e exerce a sua cidadania (LASTREs In MACIEL e Albagd li, 2007, p. 185). Assim, as 
organizações, na busca por uma maior competitividade e eficiência, passam cada vez mais a usar novas práticas de GC associadas ao crescente volume de informações e conhecimentos para a cooperação, produção e comercialização de bens e serviços.

0 contexto atual exige das organizações públicas de saúde uma abordagem diferente no atendimento ao cidadão, a qual contemple uma terapêutica integralizada, ou seja, em que saúde e doença sejam conceitos dinâmicos e a promoção da saúde desempenhe papel primordial. Os serviços públicos de saúde fundamentados no modelo biomédico ${ }^{1} \mathrm{e}$ hospitalocêntrico estão sendo duramente questionados na maneira como vêm funcionando atualmente. Assim, percebese que este modelo com uma visão especializada e focal do processo saúdedoença está ultrapassado. Torna-se fundamental, portanto, a integração das áreas de conhecimento das ciências humanas e biológicas na tentativa de explicar as necessidades humanas de saúde do indivíduo, que deve ser capaz de reconhecer-se como sujeito desse processo integral. Nesse aspecto, questiona-se a inserção excessiva das máquinas e procedimentos onerosos na terapêutica. Portanto, a incorporação de altas tecnologias não significa, necessariamente, uma melhor qualidade de vida para a população assistida ${ }^{2}$. A humanização pode ser observada como uma categoria central nas formas de melhorar 0 atendimento ao cidadão, que normalmente enfrenta longas filas, não recebe 0 devido esclarecimento do médico quanto ao seu problema de saúde e fica sem os medicamentos prescritos. As organizações de saúde, ao longo do tempo, são marcadas por relações de controle burocrático, pelafalta de recursos (humanos, financeiros, entre outros) e pelo não reconhecimento de gestores dos fatores de caráter subjetivo envolvidos nas práticas assistenciais. Assim, tal contexto tende a propiciar uma estrutura institucional caracterizada pela rigidez hierárquica, falta de comunicação entre subalternos e dirigentes e por uma disciplina autoritária. As consequiências sobre as pessoas envolvidas diretamente no trabalho da prestação do serviço de saúde são graves e normalmente acarretam a diminuição do compromisso com 0 atendimento ao cidadão (SÃo PAULo, 2003).

Diante de um cenário que indicava a necessidade de uma mudança do modelo de atenção hospitalar, foi criada pelo Ministério da Saúde, em 2003, a Política Nacional de Humanização (PNH) com 0 propósito de promover a integralidade das ações de saúde no âmbito da atenção e gestão de forma indissociável, bem como favorecer a universalidade do atendimento e 0 aumento da eqüidade por meio da utilização de novas tecnologias e especializações de saberes sem desvalorizar os processos já instituídos. D essa forma, a PNH foi idealizada como uma política transversal às demais políticas e ações de saúde e tem procurado vincularse a todos os processos em curso, assim como à elaboração de políticas de saúde por meio dos princípios orientadores da humanização. A PNH tem como objetivos: (i) reduzir as filas e o tempo de espera, com ampliação do acesso e atendimento acolhedor e resolutivo, baseado em critérios de risco; (ii) assegurar que todos os usuários do SUS conheçam os profissionais que cuidam de sua saúde e que os serviços de saúde se responsabilizem por sua referência territorial; (iii) garantir aos usuários o acesso às informações e a presença de acompanhante de 
sua livre escolha em todos os momentos do cuidado de sua saúde, bem como os demais direitos dos usuários do SUS; e (iv) consolidar nas unidades de saúde a gestão participativa dos seus trabalhadores e usuários, assim como educação permanente aos trabalhadores de saúde (BRAsIL, Ms, 2003).

Mediante o quadro atual em que se encontram algumas organizações públicas da saúde, vale questionar qual deveria ser o modelo de G estão do Conhecimento a ser empregado nessas organizações. 0 processo de humanização possui vários aspectos abordados pela GC. Ao ser adotada por uma organização de saúde, a G estão do Conhecimento pode assegurar 0 estabelecimento de um sistema de atendimento mais humanizado. Existe uma interrelação na maioria das práticas adotadas pelo processo de humanização instituído pelo Serviço Único de Saúde - SUS e pela G estão do Conhecimento, conforme mostra o Quadro 1.

Além disso, observa-se que a GC é mais abrangente e considerada a base para que as organizações de saúde possam efetivamente estabelecer o processo de humanização, uma vez que estarão focadas no desenvolvimento de uma cultura organizacional voltada à inovação e aprendizado contínuo, comprometida com os resultados de longo prazo. Segundo Stewart (2002, p. 376), um dos pontos importantes abordados pela GC é a valorização do capital humano. Assim, no ambiente organizacional as pessoas devem ser motivadas e valorizadas pelo seu trabalho por meio das recompensas institucionalizadas. A identificação ea adoção de práticas gerenciais que contemplam esses elementos são indispensáveis à atuação dos profissionais da saúde, que, cada vez mais no dia-a-dia, se deparam com situações de stress e que precisam dar respostas rápidas às crescentes demandas sociais.

Este estudo sobre a Gestão do Conhecimento procura sugerir alternativa de solução às deficiências apontadas na área da saúde e servir de instrumento capaz de identificar o conjunto de boas práticas de GC adotadas pelas organizações públicas de saúde.

\section{Modelo de Excelência em Gestão Pública}

Para permitir que a administração pública do Brasil se tornasse mais eficiente e oferecesse ao cidadão mais serviços com maior qualidade, foi proposto em 19960 Programa da Q ualidade e Participação na Administração Pública - Q PAP, com foco na gestão por resultados. Assim, o Q PAP, compreendendo a necessidade de que 0 setor público brasileiro precisava de um novo modelo de gestão pública focado em resultados e orientado para o cidadão, em substituição ao modelo burocrático, fez a adoção e a adaptação do Modelo de Excelência já utilizado para o Prêmio Nacional da Qualidade - PNQ, prêmio esse usado até os dias atuais pelo Programa Nacional de Gestão Pública e Desburocratização - Gespública.

0 Modelo de Excelência em G estão Pública é a representação de um sistema gerencial constituído por sete critérios, que orientam a adoção de práticas de gestão na organização com a finalidade de levar as organizações públicas brasileiras a padrões de desempenho reconhecidos pela sociedade e da excelência na sua gestão (BRAsiL, MP, 2006, p. 7). A representação gráfica do Modelo de Excelência da Figura 1 mostra a relação entre os sete critérios da avaliação da gestão pública, enfatizando que devem estar alinhados às estratégias da organização. 


\section{Q uadro 1: Caracteństicas da Gestão Humanizadora e da GC}

\begin{tabular}{|c|c|}
\hline $\begin{array}{l}\text { Principais características do processo de } \\
\text { humanização na área de saúde }\end{array}$ & $\begin{array}{l}\text { Principais características do processo da } \\
\text { Gestão do Conhecimento }\end{array}$ \\
\hline $\begin{array}{l}\text { Aumento do grau de co-responsabilidade dos } \\
\text { diferentes atores envolvidos, implicando a } \\
\text { mudança da cultura da atenção dos usuários e } \\
\text { da gestão dos processos de trabalho. }\end{array}$ & $\begin{array}{l}\text { Papel indispensável da alta administração na } \\
\text { definição dos campos de conhecimentos onde } \\
\text { a organização deve focalizar seus esforços de } \\
\text { aprendizado. }\end{array}$ \\
\hline $\begin{array}{l}\text { A humanização focaliza com especial atenção } \\
\text { os processos de trabalho e os modelos de } \\
\text { gestão e de planejamento. }\end{array}$ & $\begin{array}{l}\text { D esenvolvimento de uma cultura organi- } \\
\text { zacional voltada à inovação e aprendizado } \\
\text { contínuo, comprometida com os resultados } \\
\text { de longo prazo. }\end{array}$ \\
\hline $\begin{array}{l}\text { G estão participativa entre funcionários e } \\
\text { chefias requerendo formas de relação institu- } \\
\text { cional mais humanizada e um clima organiza- } \\
\text { cional mais harmonioso. }\end{array}$ & $\begin{array}{l}\text { Novas estruturas organizacionais baseadas no } \\
\text { trabalho de equipes multidisciplinares com } \\
\text { alto grau de autonomia. }\end{array}$ \\
\hline $\begin{array}{l}\text { Estabelecimento de um processo contínuo de } \\
\text { troca de informações e de experiências nos } \\
\text { programas do SUS, por meio do trabalho em } \\
\text { rede entre seus vários setores. }\end{array}$ & $\begin{array}{l}\text { Novas práticas e políticas de gestão de pessoas } \\
\text { associadas à aquisição de conhecimentos } \\
\text { externos einternosà organização, assim como } \\
\text { à geração, difusão e armazenamento do } \\
\text { conhecimento interno. }\end{array}$ \\
\hline $\begin{array}{l}\text { Fortalecimento do trabalho em equipe } \\
\text { multiprofissional, estimulando a transdisci- } \\
\text { plinaridade e a grupalidade. Avaliação de re- } \\
\text { sultados do processo de humanização por } \\
\text { meio da análise das dificuldades e erros; identi- } \\
\text { ficação de oportunidades de melhoria dos } \\
\text { processos organizacionais; e pesquisa de } \\
\text { satisfação de profissionais e usuários. }\end{array}$ & $\begin{array}{l}\text { Manutenção de um ambiente organizacional } \\
\text { de elevada confiança, transparência e colabo- } \\
\text { ração de modo a favorecer o aprendizado } \\
\text { individual e coletivo. } \\
\text { Esforços de mensuração dos resultados refe- } \\
\text { rentes aos ativos representados pelo conheci- } \\
\text { mento organizacional. }\end{array}$ \\
\hline $\begin{array}{l}\text { Garantia das condições de infra-estrutura } \\
\text { para que os profissionais efetuem seu traba- } \\
\text { lho de modo digno e criativo e possam parti- } \\
\text { cipar como co-gestores do processo de } \\
\text { trabalho. }\end{array}$ & $\begin{array}{l}\text { Processos de aprendizado com o ambiente, } \\
\text { em particular por meio de alianças com outras } \\
\text { organizações e de estreitamento do relaciona- } \\
\text { mento com os seus usuários/ clientes. }\end{array}$ \\
\hline
\end{tabular}

Fonte: Elaboração própria a partir das informações do Programa Nacional de Humanização da Assistência Hospitalar - PNHAH (BRASIL, Ministério da Saúde, 2001) e de Terra (2001).

Em 1998 foi criado o Prêmio da Q ualidade do Governo Federal (PQGF) com o objetivo principal de estimular as organizações do setor público a implementarem programas de melhoria do desempenho institucional e reconhecer formalmente os resultados das instituições comprometidas com a implementação da Gestão Pública pela Qualidade. O programa QPAP teve o seu nome mudado para Programa de Qualidade no Serviço Público (PQ SP) em 1999, cujo foco era a satisfação do cliente. Em 2005, houve a fusão dos Programas da Qualidade no Serviço Público (PQSP) e de D esburocratização, originando o atual Programa Nacional de Gestão Pública e Desburocratização - Gespública. 


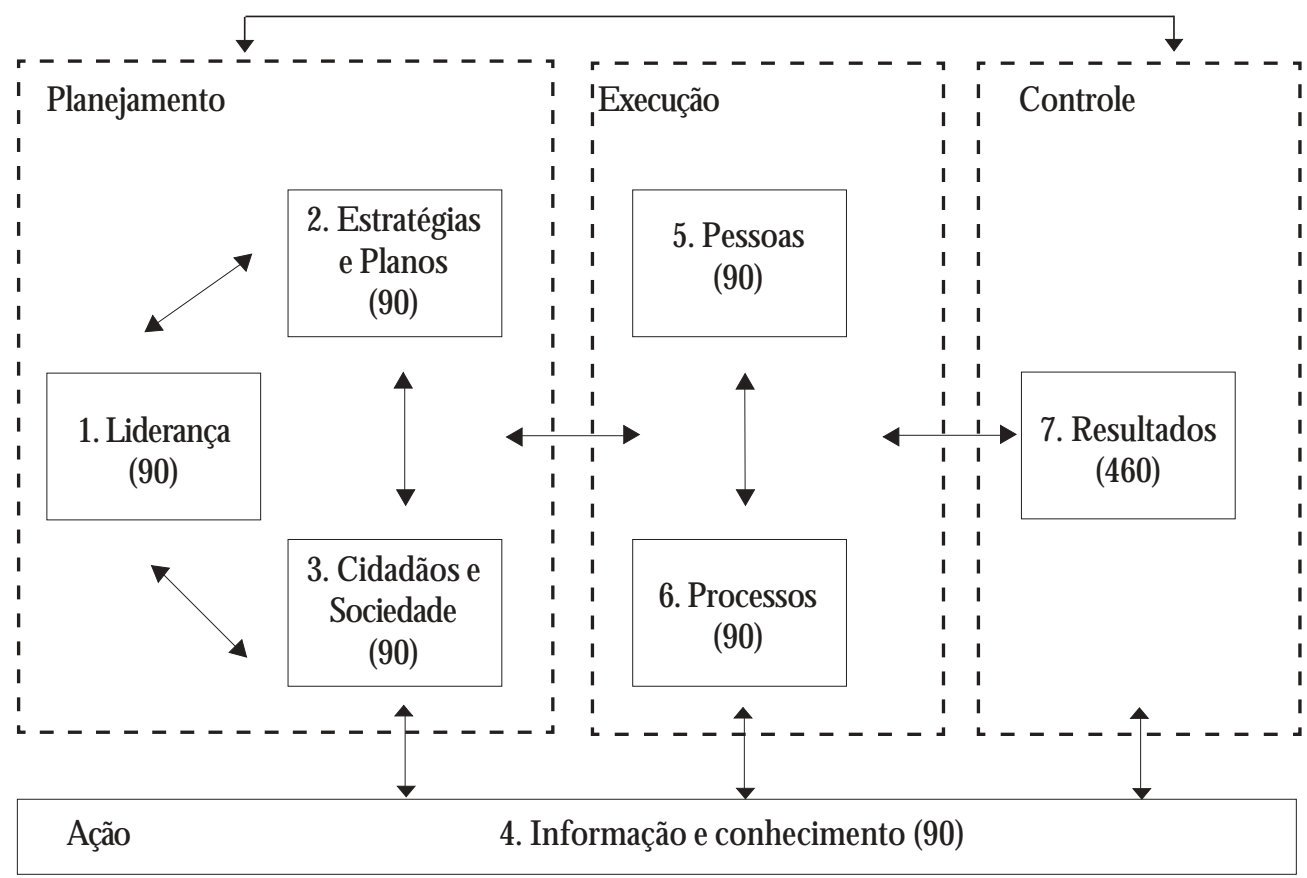

Legenda: A pontuação máxima para cada critério encontra-se entre parênteses.

Fonte: BRASIL, MP, 2006.

\section{Figura 1: Representação Gráfica entre os Elementos de Gestão da Organização.}

Metodologia de Avaliação das Práticas de Gestão nas 0 rganizações Públicas de Saúde

$\mathrm{O}$ presente estudo utilizou-se do item $4.3 \mathrm{G}$ estão do Conhecimento, do Critério 4 do Modelo de Excelência em Gestão Pública do Gespública na elaboração das assertivas da terceira parte do questionário submetido às organizações públicas de saúde para avaliar suas práticas de gestão. O item 4.3 aborda como o conhecimento dentro das organizações públicas é identificado, desenvolvido, cultivado, protegido e compartilhado, e é formado por cinco alíneas (requisitos). 0 questionário aplicado nas organizações abrangidas pelo estudo foi elaborado utilizando-se as assertivas das alíneas A, B, C e D do Modelo de Excelência em Gestão Pública, conforme mostra o Quadro 2 a seguir, e possui a característica investigativa, uma vez que procura responder às questões específicas referentes ao objetivo da pesquisa (COOPER e Schind LeR, 2003, p. 274).

0 questionário foi montado sem a alínea E por ser de conhecimento prévio 0 fato de quase todas as organizações pesquisadas não terem implementado sistematicamente a Gestão do Conhecimento. 0 universo da pesquisa abrangeu 30 organizações de saúde que responderam ao questionário, ou seja, 11 organizações de saúde que fizeram adesão ao Gespública pertencentes à administração pública no nível Federal, Estadual e Municipal e 19 organizações de saúde (Centros de Pesquisas Clínicas) indicadas pelo Departamento de Ciência e Tecnologia (Decit) do Ministério da Saúde. 
Quadro 2: Questionário-orientador

\begin{tabular}{|c|c|c|c|}
\hline Alineas & Perguntas & $\begin{array}{l}\text { Descriçlo da } \\
\text { evidtincia ebjetiva } \\
\text { da prática de } \\
\text { zestäo }\end{array}$ & $\begin{array}{l}\text { Descriçlo das } \\
\text { eportunidades } \\
\text { de melheria }\end{array}$ \\
\hline $\mathbf{A}$ & 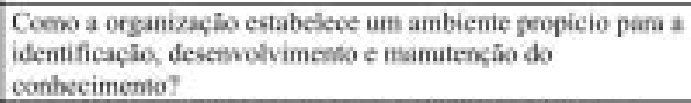 & & \\
\hline AI & $\begin{array}{l}\text { Camo a organizaclio incentiva o pensamenko criative e } \\
\text { inovadar? }\end{array}$ & & \\
\hline$\Delta 2$ & $\begin{array}{l}\text { Coeno a organizaçio faz a atracio ou relensio de talemos e } \\
\text { compcténcias? }\end{array}$ & & \\
\hline B & Como č protegiko o conhesiencnto" & & \\
\hline $\mathbf{C}$ & 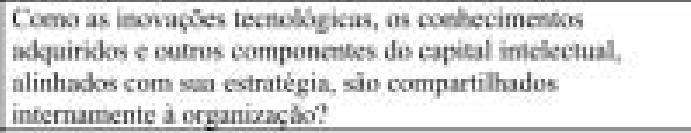 & & \\
\hline $\mathbf{D}$ & $\begin{array}{l}\text { Como a organizasiono assegura que a gestio de conbccimenio } \\
\text { seja utilixada para melhorar os scus produtes e servicos? }\end{array}$ & & \\
\hline
\end{tabular}

Fonte: Elaboração própria adaptada de BRASIL, MP, 2006, p.31.

O Modelo de Excelência em G estão Pública do Gespública foi colocado como um guia para avaliar as práticas de gestão apontadas pelas organizações públicas de saúde pesquisadas. A metodologia utilizada nesta pesquisa referente às respostas do questionário foi a mesma usada pelo Gespública para avaliar as organizações públicas. Foram consideradas como práticas, ou processos, ou métodos, ou metodologias de gestão as atividades executadas sistematicamente com a finalidade de gerenciar uma organização, consubstanciadas nos padrões de trabalho ${ }^{4}$ estabelecidos (BRAsiL, MP, 2006, p. 57).

A metodologia usada pelo Modelo de Excelência propõe que, para se alcançar excelência do desempenho, as práticas de gestão relacionadas aos critérios sejam avaliadas nas três dimensões: M étodo, E x tensão da A plicação e Resultados, conforme 0 Quadro 3.

\section{Q uadro 3: Dimensões e Fatores de Avaliação do Gespública}

\begin{tabular}{|l|l|}
\hline Dimensões & Fatores deAvaliação \\
\hline Método & $\begin{array}{l}\text { - Adequação } \\
\text { - Proatividade } \\
\text { - Refinamento } \\
\text { - Inovação }\end{array}$ \\
\hline Aplicação & $\begin{array}{l}\text { - Disseminação } \\
\text { - Continuidade }\end{array}$ \\
\hline Resultados & $\begin{array}{l}\text { Relevância } \\
\text { - Desempenho } \\
\text { - Tendência }\end{array}$ \\
\hline
\end{tabular}

Fonte: Brasil, Ministério do Planejamento, 2001, p. 77. 
A dimensão M étodo se refere aos métodos e aos processos utilizados para executar as atividades solicitadas pelos itens de cada critério. Quanto ao método, as práticas de gestão podem ser avaliadas como A dequação; Proatividade; Refinamento e Inovação. A A dequação é relativa ao atendimento dos requisitos do item 4.3, anteriormente referido, conforme o perfil da organização de saúde. As práticas de gestão Proativas são aquelas com a capacidade de se antecipar aos fatos com ações preventivas e que são capazes de promover a inovação e a melhoria dos processos, serviços e produtos de saúde. O Refinamento diz respeito ao estágio avançado de evolução da prática de gestão, alcançado pela aplicação sistemática e continuada do aprendizado. As práticas de gestão Inovadoras são caracterizadas como inéditas ou incomuns no setor de saúde (BRASIL, MP, 2006, p. 49).

Já a Extensão da Aplicação guarda relação com a disseminação horizontal e vertical do método dos itens pelos principais processos, setores e unidades da organização e à sistematização da utilização desse método. Portanto, a aplicação do método é avaliada segundo os fatores D isseminação e C ontinuidade. A avaliação da disseminação de uma prática de gestão refere-se ao grau de adoção nas áreas, nos serviços e nos processos pertinentes à organização de saúde. Por sua vez, a continuidade de uma prática de gestão referese ao grau de sua utilização de maneira periódica e ininterrupta.

Finalmente, a dimensão Resultados está associada às conseqüências da aplicação do método, em que são analisados os níveis atuais de desempenho da organização, as tendências existentes e a consistência do desempenho e se, efetivamente, esses níveis e tendências decorrem dos métodos aplicados aos principais processos, setores e unidades da organização.

No Modelo de Excelência, a organização é pontuada quanto ao estágio em que esta se encontra em relação ao atendimento dos critérios de excelência, cuja pontuação máxima de cada um está mostrada na Figura 1. Os critérios de 1 a 6 são avaliados em um percentual conforme Tabela de Pontuação para Métodos e Aplicação, considerando-se os fatores Adequação e

Exemplaridade relacionados com o Método e os fatores Continuidade, bem como Disseminação, com a $\mathrm{E}$ x tensão da A plicação.

0 critério 7 é avaliado conforme um percentual descrito na Tabela de Pontuação de Resultados, a partir dos seguintes fatores: Relevância, Desempenho e Tendência dos Resultados. A composição da pontuação dos sete critérios gerará a pontuação global, que se enquadrará em 
uma das cinco faixas de pontuação referentes ao grau da gestão em que a organização se encontra.

Assim, o processo de avaliação permite à organização tanto identificar os pontos fortes e as oportunidades de melhoria de de resposta é prevista entre 40\% e 50\% (Cooper e Schindler, 2003). As organizações foram avaliadas individualmente em cada uma das alíneas A,B,C e D, conforme exemplificado no Quadro 4.

\section{Q uadro 4: Exemplo da avaliação das respostas da alínea A}

\begin{tabular}{|c|c|c|c|c|c|}
\hline \multirow{2}{*}{$\begin{array}{l}\text { Nome da } \\
\text { Organizaşâo }\end{array}$} & \multirow{2}{*}{$\begin{array}{l}\text { Caracteristica } \\
\text { da arganixasẩo } \\
\text { participante } \\
\text { e becal }\end{array}$} & \multicolumn{3}{|c|}{ Pontes Fotic: } & \multirow[b]{2}{*}{$\begin{array}{l}\text { Oportunidades } \\
\text { de mellharia }\end{array}$} \\
\hline & & $\begin{array}{l}\text { Descriçîno das } \\
\text { prúticas de gestio }\end{array}$ & $\begin{array}{c}\text { Mitiodo } \\
\text { (Adequacio/Pro- } \\
\text { atividade/ } \\
\text { Refinamento/ } \\
\text { Inovacio) }\end{array}$ & $\begin{array}{l}\text { Aplicaçĩo } \\
\text { (Disseminaşa/o/ } \\
\text { Continuidade) }\end{array}$ & \\
\hline Oryanizscasis $\mathrm{X}$ & $\begin{array}{l}\text { Organizaçlo } \\
\text { perteneente } \\
\text { ao Gespublica } \\
\text { (Porto Alegre - } \\
\text { RS). }\end{array}$ & $\begin{array}{l}\text { A organizasio prosui } \\
\text { um Fundo de } \\
\text { Incentivo a Pesquisa } \\
\text { (FIPE) que destina } \\
\text { 0,08\% do seu } \\
\text { faturamento para o } \\
\text { fomento à pesquisa. }\end{array}$ & Adecposta. & Desseminada. & 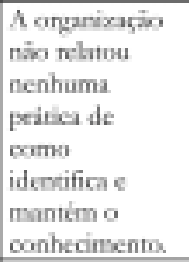 \\
\hline Organizacào $\mathrm{Y}$ & $\begin{array}{l}\text { Centros de } \\
\text { Pesquisa } \\
\text { Clinica } \\
\text { (Belem, PA). }\end{array}$ & $m$ & $m$ & & $\begin{array}{l}\text { Niko possui } \\
\text { nenhuma } \\
\text { peitica de } \\
\text { pretiak }\end{array}$ \\
\hline
\end{tabular}

Fonte: Elaboração própria dos autores.

sua gestão frente aos critérios de excelência, como planejar melhorias internas de suas práticas gerenciais que conduzam ao maior desempenho institucional. D essa forma, as respostas da terceira parte dos questionários somente foram avaliadas quanto ao Método e Aplicação de suas práticas de gestão para atenderem aos requisitos solicitados do referido item 4.3. Não se pretendeu analisar os resultados, já que nenhuma informação quantitativa foi solicitada aos respondentes.

\section{Resultados}

0 número de respondentes do questionário foi de $58 \%$ para os Centros de Pesquisas Clínicas e de 45\% para as organizações pertencentes ao Gespública, constituindo uma boa taxa de retorno segundo a literatura especializada, cuja taxa
Para cada organização foi possível avaliar as práticas de gestão relativas à GC no que se refere às duas dimensões seguintes: método edisseminação. Quanto ao método, não foi possível em nenhuma das práticas apresentadas verificar o grau de refinamento, pois não se informou nenhum aprendizado sobre elas. Como as organizações não descreveram as suas práticas detalhadamente, a análise limitou-se aos seguintes fatores: adequação, proatividade e inovação. Essas também não relataram quais padrões de trabalho e controles são utilizados para verificar se as práticas estão sendo executadas conforme o previsto. Quanto à dimensão aplicação, não foi informada a data de implementação da maioria das práticas apresentadas, o que dificultou saber o grau de continuidade. Assim, aparecem poucas práticas contínuas, 
pois só se pode afirmar sobre a continuidade da prática mediante a explicitação do seu início. Quanto à disseminação, foi considerado que todas as práticas em princípio foram disseminadas pela organização, a não ser quando ficou evidente que as mesmas não estavam ainda disseminadas pelos setores ou departamentos. Esse tipo de análise permite mostrar as práticas de gestão tidas como exemplares e que podem ser seguidas por outras organizações de saúde, bem como as deficiências gerenciais descritas como oportunidades de melhoria. Nos Quadros 5 e 6, descreve-se para cada fator avaliativo a quantidade de práticas relativas a cada alínea do questionário referente às organizações adesas ao
Gespública e aos Centros de Pesquisas Clínicas.

Ao comparar os Quadros 5 e 6, podese constatar que as organizações adesas possuem uma taxa maior de práticas adequadas, proativas, inovadoras, disseminadas e com continuidade do que os Centros de Pesquisas Clínicas. Com relação à taxa de práticas não adequadas ao requisito solicitado, pode-se considerar que ambas possuem o mesmo grau de não atendimento: 0,9 e 0,7 respectivamente.

Outro aspecto importante é que as organizações adesas ao Gespública são mais proativas e inovadoras do que os Centros de Pesquisas Clínicas, mesmo sendo nessas últimas organizações onde

\section{Q uadro 5: Q uantidade de práticas apresentadas por fator avaliativo em relação às organizações adesas ao Gespública}

\begin{tabular}{|c|c|c|c|c|c|c|c|}
\hline \multirow{2}{*}{$\begin{array}{l}\text { Fatcones de } \\
\text { Avaliacilo }\end{array}$} & \multicolumn{7}{|c|}{ Nimems de priticas } \\
\hline & A & A1 & $\mathrm{A} 2$ & B & C & D & $\begin{array}{l}\text { Total do Fatoe / } \mathrm{N}^{*} \text { de } \\
\text { Organizacồes Adesas } \\
\text { Respondentes }\end{array}$ \\
\hline Adequada & 7 & 5 & 4 & 5 & 8 & 2 & 3.4 \\
\hline Prostivalade & 3 & 1 & 1 & 3 & 1 & 1 & 1,1 \\
\hline Inovadoes & 0 & 2 & 0 & 4 & 2 & 1 & 1,0 \\
\hline Disscminada & 7 & 5 & 4 & 5 & 8 & 2 & 3.4 \\
\hline Concinuidade & 1 & 1 & 0 & 1 & 0 & 0 & 0.3 \\
\hline Nilo adequada & 0 & 2 & 2 & 1 & 1 & 2 & 0.9 \\
\hline
\end{tabular}

Fonte: Elaboração dos autores.

Legenda: A, A1, A2, B, C e D são as alíneas que contêm os requisitos do item avaliado.

\section{Q uadro 6: Quantidade de práticas apresentadas por fator avaliativo em relação aos centros de Pesquisa Clínica}

\begin{tabular}{|c|c|c|c|c|c|c|c|}
\hline \multirow{2}{*}{$\begin{array}{l}\text { Fabcines de } \\
\text { Avalixazio }\end{array}$} & \multicolumn{7}{|c|}{ Nümeto de Práticas } \\
\hline & A & A1 & $A 2$ & B & C & D & $\begin{array}{l}\text { Toul do Fatoe / Nümero de Centros de } \\
\text { Pesprowas Clinicas Resporstentes }\end{array}$ \\
\hline Adequada & 4 & 8 & 6 & 5 & 8 & 2 & 30 \\
\hline Proatividade & 0 & 1 & 0 & 3 & 1 & 1 & 0.5 \\
\hline Inowadona & 1 & 0 & 0 & 3 & 1 & $\theta$ & 0.4 \\
\hline Disseminasta & 4 & 6 & 6 & 4 & 8 & 2 & 27 \\
\hline Continuidade & 0 & 0 & 0 & 0 & 0 & 0 & 0 \\
\hline Nïs adequads & 3 & 1 & 2 & 1 & 0 & 1 & 07 \\
\hline
\end{tabular}

Fonte: Elaboração dos autores.

Legenda: A, A1, A2, B, C e D são as alíneas que contêm os requisitos do item avaliado. 
predomina a cultura da pesquisa e do desenvolvimento científico. Isso mostra que uma organização, para ser inovadora na sua gestão, tem que estimular um ambiente propício ao pensamento criativo e inovador.

No Quadro 7, foi resumido o nível de atendimento de cada organização adesa para com cada requisito solicitado pelo questionário. Assim, pode-se verificar que duas organizações possuem práticas de gestão que atendem plenamente a todos os requisitos, enquanto somente três possuem poucas práticas que respondem ao que foi solicitado. Isso mostra que 0 fato de a organização ser adesa ao Gespública não permite presumir que ela esteja aplicando na sua gestão o Modelo de Excelência. Se considerarmos as organizações acima de $50,0 \%$ no grau de atendimento aos requisitos, percebe-se que a alínea $\mathrm{D}$ é a que apresenta maior número de organizações que não possuem práticas.

Quanto aos Centros de Pesquisas Clínicas, verifica-se no Quadro 8 que nenhuma organização pesquisada atende completamente a todos os requisitos. A maioria das organizações pesquisadas atendeu aos requisitos A1 e C. Isso é devido ao fato de tais requisitos estarem ligados diretamente à missão intrínseca de cada organização, qual seja a pesquisa.

Pode-se verificar que um grande número de Centros de Pesquisas Clínicas não atende ao requisito solicitado na alínea D do mesmo modo que as organizações adesas. Isso mostra uma dificuldade, por parte das organizações, em implementar práticas capazes de assegurar que a GC seja utilizada com intuito de melhorar os serviços.

\section{Análises críticas}

As organizações pesquisadas foram classificadas quanto ao estágio em que se encontram em relação ao item 4.3 do Modelo de Excelência, tendo como parâmetro aquelas que atendem a mais de $80 \%$ dos requisitos, conforme mostrado nos Q uadros 7 e 8. Na descrição do estágio em que se encontra a organização, foi considerado o conjunto de práticas que cada uma possui para atender aos requisitos do item 4.3 do Modelo de Excelência. D essa forma, as organizações podem ter uma idéia de quanto precisam

\section{Q uadro 7: Síntese das práticas apresentadas pelas organizações adesas ao Gespública}

\begin{tabular}{|c|c|c|c|c|c|c|c|}
\hline \multirow[t]{2}{*}{ Orğanixaçān } & \multicolumn{6}{|c|}{ Alinea $\varepsilon$ Práticas de Geatân } & \multirow{2}{*}{$\begin{array}{c}\text { \% de Requisitos } \\
\text { Atendidos }\end{array}$} \\
\hline & $\boldsymbol{\Lambda}$ & A1 & 12 & B & c & $\mathrm{D}$ & \\
\hline HCPA & $\mathbf{T}$ & NT & NT & $\mathrm{T}$ & $\mathrm{T}$ & NT & 50,0 \\
\hline Hospital FEmina & NT & $\mathbf{T}$ & $\mathbf{T}$ & NT & $\mathrm{T}$ & NT & 50,0 \\
\hline Instituso de Tecnologria de Imamobioléricus & NT & $T$ & $\mathbf{T}$ & $\mathrm{T}$ & $\mathrm{T}$ & NT & 66,7 \\
\hline Indiruto Estadual de Hematologia A. S. C. & $\mathbf{T}$ & $\mathbf{T}$ & $\mathbf{T}$ & $\mathrm{T}$ & $\mathrm{T}$ & $\mathbf{T}$ & 160,0 \\
\hline Instituto Nacional do Cäncer & $\mathbf{T}$ & $\mathrm{T}$ & NT & $\mathrm{T}$ & $\mathrm{T}$ & NT & $60, ?$ \\
\hline Laboratório Quimico Farmac. do Exército & $T$ & $\mathbf{T}$ & $\mathbf{T}$ & $\mathbf{T}$ & $\mathrm{T}$ & $\mathrm{T}$ & 100,0 \\
\hline Fund, de Hematología & $\mathrm{T}$ & NT & NT & NT & $\mathrm{T}$ & Nit & 33,3 \\
\hline Huspital Geral de Salvador & $\mathbf{T}$ & NT & NT & NT & $\mathrm{T}$ & NT & 33,3 \\
\hline Hospital da Criansa Cnnceiçân & $\mathbf{T}$ & NT & NT & NT & NT & NT & 16,7 \\
\hline
\end{tabular}

Fonte: Elaboração própria dos autores.

Legenda: $\mathrm{NT}=$ Não tem nenhuma prática de gestão; $\mathrm{T}=$ Tem pelo menos uma prática de gestão que atenda ao requisito; HCPA = Hospital das Clínicas de Porto Alegre; HEMO BA = Fundação de Hematologia e Hemoterapia da Bahia. 


\section{Quadro 8: Síntese das Práticas Apresentadas pelos Centros de Pesquisa Clínica}

\begin{tabular}{|c|c|c|c|c|c|c|c|}
\hline \multirow[t]{2}{*}{ Ongansaçio } & \multicolumn{6}{|c|}{ Ninea e Praticas de Giestion } & \multirow{2}{*}{$\begin{array}{l}\text { \% de Requisitos } \\
\text { Atendidas }\end{array}$} \\
\hline & $A$ & $A 1$ & $A 2$ & B & C & D & \\
\hline $\begin{array}{l}\text { Cenero de Pesquisa Cinica da Faculdade de } \\
\text { Medicina da USP. }\end{array}$ & NT & $\mathrm{T}$ & $\mathbf{T}$ & $\mathbf{T}$ & $\mathbf{T}$ & $\mathbf{T}$ & 63,3 \\
\hline $\begin{array}{l}\text { Uniade de Pesquisa Clinica do Hospital das } \\
\text { Clinicas -UFG }\end{array}$ & NT & NT & $\mathbf{T}$ & NT & $\mathbf{T}$ & NT & 33,3 \\
\hline Huspical Leninersiniros I. ck Barros Barreto & NT & $\mathrm{T}$ & $\mathbf{T}$ & $\mathbf{T}$ & $\mathbf{T}$ & $\mathrm{T}$ & 433 \\
\hline Cenrro de Pesquisas Clinicas do IILWC - FCPC & NT & NT & NT & NT & NT & NT & 09 \\
\hline $\begin{array}{l}\text { Uninde de Resquisa Clínica des Hospitais de } \\
\text { Ensino HLOC E PROCAPE - LAFUPE }\end{array}$ & $\mathrm{T}$ & NT & $\mathbf{T}$ & $\mathbf{T}$ & $\mathbf{T}$ & NT & 66,7 \\
\hline Cestro de Pesuuisa do liUUFMA & $T$ & $\mathrm{~T}$ & NT & NT & $\mathbf{T}$ & NT & 500 \\
\hline Cenero de Peseuisa Clinica - IMIP/PE & $T$ & $\mathrm{~T}$ & $\mathbf{T}$ & NT & $\mathbf{T}$ & NT & 66,7 \\
\hline Unidade de Pesquisa Cínica do HUCFF/UFRI & NT & $T$ & NT & NT & NT & NT & 16,7 \\
\hline $\begin{array}{l}\text { Dinatude de Resquisa Clinica Saide da Mulher e } \\
\text { Cranga/IIF }\end{array}$ & $\mathrm{T}$ & $\mathrm{T}$ & NT & $\mathbf{T}$ & $\mathbf{T}$ & NT & 66,7 \\
\hline 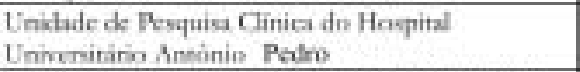 & NT & $\mathrm{T}$ & NT & $\mathbf{T}$ & NT & NT & 500 \\
\hline Unistade de Pesquisa Clinica do HC.MRP -USP & NT & $T$ & $\mathbf{T}$ & NT & $\mathbf{T}$ & NT & 500 \\
\hline
\end{tabular}

Fonte: Elaboração dos autores.

Legenda: $\mathrm{NT}=$ Não possui nenhuma prática de gestão. $\mathrm{T}=$ Possui pelo menos uma prática de gestão que atenda ao requisito. UFG = Universidade Federal de Goiás; HUOC $=$ Hospital Universitário O swaldo Cruz; HUWC = Hospital Universitário Walter Cantídio; HUUFMA = Hospital Universitário da Universidade Federal do Maranhão; IMIP/ PE = Instituto Materno Infantil Prof. Fernando Figueira; HUCFF/ UFRJ = Hospital Universitário Clementino Fraga Filho; HCMRP-USP = Hospital das Clínicas da Faculdade de Medicina de Ribeirão Preto.

melhorar a sua gestão para alcançar um estágio mais evoluído em relação à Gestão do Conhecimento.

Todas as organizações pesquisadas que estão no Estágio Inicial mostraram práticas de gestão não sistematizadas e essencialmente reativas. No Quadro 9, observa-se um total de quatro organizações: duas adesas ao Gespública e dois Centros de Pesquisas Clínicas, os quais se encontram num estágio mais avançado em relação à Gestão do Conhecimento e, conseqüentemente, possuem maior capacidade para agir e inovar.

Existe a percepção de que a maioria das organizações pesquisadas desconhece a ligação entre a Gestão do Conhecimento e o processo de humanização propagado pelo SUS/ MS, por isso não existe uma preocupação estratégica para a sua adoção.

\section{Conclusões e recomendações}

O estudo mostrou que a maioria das organizações pesquisadas está em um estágio inicial de atendimento aos requisitos do item 4.3 referente à G estão do Conhecimento do Modelo de Excelência em Gestão Pública.

No Brasil, processos recentes de formulação de políticas de Ciência, Tecnologia e Inovação (CT\&I) e de Informação e Informática em Saúde (IIS) têm procurado a integração de esforços de diversos atores em torno de agendas que incluem todos os aspectos relacionados à informação relevante na determinação e condicionamento dos níveis de saúde dos indivíduos, com a finalidade de ajudar na tomada de decisões relativas à atenção e à gestão, bem como no 


\section{Q uadro 9: Classificação das organizações quanto ao grau de atendimento aos requisitos}

\begin{tabular}{|c|c|c|c|c|}
\hline \multirow[t]{2}{*}{ Eatiggio } & \multirow{2}{*}{$\begin{array}{l}\text { Grau de } \\
\text { stendimento aos } \\
\text { requisitos do } \\
\text { item } 4.3\end{array}$} & \multirow{2}{*}{$\begin{array}{l}\text { Descrição do estágio ent que \$e } \\
\text { encontra a organixaçio }\end{array}$} & \multicolumn{2}{|c|}{ Nümeto de Orgranixaçōes } \\
\hline & & & $\begin{array}{l}\text { Organizaçöes } \\
\text { Adesaas ao } \\
\text { Gexpública }\end{array}$ & $\begin{array}{l}\text { Centro de } \\
\text { Pesquisas } \\
\text { Clinicas }\end{array}$ \\
\hline Avançado & $\begin{array}{l}\text { Maior que } 80 \% \\
\text { dos requikitos } \\
\text { atendidos }\end{array}$ & 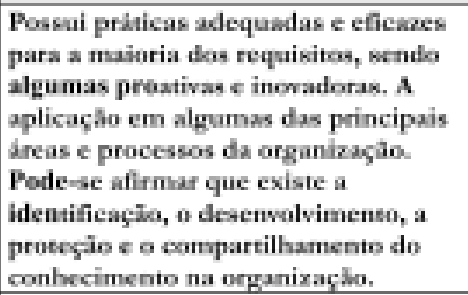 & 2 & 2 \\
\hline Inicial & $\begin{array}{l}\text { Menar que } 50 \% \\
\text { dos requisitos } \\
\text { atendidos }\end{array}$ & 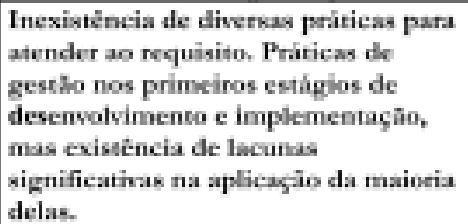 & 7 & 9 \\
\hline
\end{tabular}

Fonte: Elaboração própria dos autores.

controle sobre ações, serviços, redes e sistemas de saúde. Um novo modelo de pesquisa clínica, voltado às reais necessidades do Sistema Único de Saúde, foi instalado no País a partir de $2005 \mathrm{com}$ investimentos da ordem de $\mathrm{R} \$ 29$ milhões para 0 estabelecimento de uma rede de laboratórios, que em sua maioria participou do referido estudo. Nesse contexto e com base na pesquisa realizada com as organizações públicas de saúde, sugerese a inclusão da GC na da Política Nacional de CT\&I e na Política Nacional de Humanização (PNH) para a área da saúde como um instrumento gerencial capaz de garantir a identificação, divulgação, controle, desenvolvimento e manutenção do conhecimento.

Assim, é possível apontar algumas recomendações decorrentes do estudo, a saber:

- O Ministério da Saúde deveria estimular todas as organizações públicas de saúde a utilizarem o Modelo de Excelência em G estão Pública e alocar mais recursos para esse fim;

- As organizações só deveriam ser consideradas como adesas pelo Gespública quando realmente aplicarem na íntegra 0 Modelo de Excelência em G estão Pública;

- O Programa Gespública deveria divulgar mais os benefícios trazidos para as organizações que utilizam o Modelo de Excelência em Gestão Pública;

- Um guia explicativo sobre a G estão do Conhecimento para o serviço público deveria ser elaborado;

- Um portal virtual para troca de práticas de gestão entre as instituições adesas ao Programa Gespública poderia ser criado.

Finalizando, épreciso que o G espública elabore um caderno explicativo sobre a forma ideal de organizações relatarem suas práticas de gestão, uma vez que as organizações abrangidas pelo estudo encontraram dificuldades para relatar as suas práticas de gestão conforme a 
metodologia usada pelo Modelo de Excelência em G estão Pública.

As organizações da área da saúde, ao aplicarem a G estão do Conhecimento, estarão buscando o aprendizado organizacional e, com isso, possibilitando um processo de melhoria contínua, conforme preconiza o Modelo de Excelência em Gestão Pública. O conhecimento internalizado representa a inteligência da organização, viabilizando a análise crítica ea execução de ações necessárias em todos os níveis. Nesse sentido, a Gestão do Conhecimento constitui-se base de todo o Modelo de Excelência, pois é através do conhecimento que se torna possível o elo e a correlação entre todos os critérios e, portanto, todos os elementos gerenciais inerentes à organização.

(Artigo recebido em 3 de dezembro de 2007. Versão final em 16 de abril de 2008)

\section{Notas}

1 O modelo biomédico não vê o corpo como uma máquina perfeita, mas como uma máquina que tem ou terá problemas que só especialistas podem constatar (KoIFMAN, 2001).

2 MotтA, E. A necessidade da atenção básica na formação em saúde. Disponível em: <http:/ / www.pqsp.planejamento.gov.br/ sobre_pqsp.aspx>. Acesso em: 10 out. 2006.

3 Disponível em: <http:/ / www.pqsp.planejamento.gov.br/ sobre_pqsp.aspx?url= apresentacao.htm>. Acesso em: 12 out. 2006.

4 Padrões de trabalho são regras que orientam 0 funcionamento das práticas de gestão e podem estar notavelmente na forma de diretrizes organizacionais, procedimentos, fluxogramas e normas legais, permitindo orientar a organização na execução da prática em si (BRASIL, MP, 2006, p. 57).

\section{Referências bibliográficas}

Batista, F. F. G overno que aprende: Gestão do conhecimento em organizações do executivo federal. Brasília: Instituto de Pesquisa Econômica Aplicada/ IPEA, 2004. (Texto para D iscussão n ${ }^{0}$ 1022).

. 0 D esafio da G estão do Conhecimento nas Á reas de A dministração e Planejamento das Instituigões Federais de E nsino Superior (IFE S). Brasília: Instituto de Pesquisa Econômica Aplicada/ IPEA, 2006. (Texto para D iscussão no 1118).

BATISTA, F. F.; PACHECO F. F.; TeRRA J. C. C. G estão do conhecimento na administracão pública. Brasília: Instituto de Pesquisa E conômica Aplicada/ IPEA, 2005. (Texto para D iscussão no 1095).

BRAsIL, Ministério do Planejamento, Orçamento e G estão. Instrumento de A valiação da G estão Pública - Ciclo 2001. Brasília, D F: MP, 2001.

. Ministério da Saúde. H umaniza SU S: política nacional de humanização. Brasília: MS/ Secretaria-Executiva. Núcleo Técnico da Política Nacional de Humanização, 2003. 
Ministério da Saúde. Programa N acional de H umanização da A ssistência H ospitalar- PN H A H . Brasília, D F: Ministério da Saúde, 2001. (Série C. Projetos, Programas e Relatórios, n. 20. Secretaria de Assistência à Saúde, Programa Nacional de Humanização da Assistência Hospitalar).

. Ministério do Planejamento, Orçamento e Gestão. Instrumento deA valiação da G estão Pública - Cido 2006. Brasília, DF: MP-GESPÚBLICA/ SEGES, 2006. (Versão 1).

Cooper, D. R.; Schindler P. S. M étodos de pesquisa em administração. 7 ed. Porto Alegre: Bookman, 2003.

D avenport, T. H.; Marchand D. A.; D ickson T. D ominando a gestão da informação. Porto Alegre: Bookman, 2004.

KoIfman, L. O modelo biomédico e a reformulação do currículo médico da Universidade Federal Fluminense. H istória, Ciências, Saúde - Manguinhos, Rio de janeiro, v. VIII, n. 1, p. 48-70, mar./ jun. 2001.

LASTREs, H. M. M. Invisibilidade, injustiça cognitiva e outros desafios à compreensão da economia do conhecimento. In: Maciel, M. L.; Albagli, S. (O rg.). Informação e desenvolvimento: conhecimento, inovação e apropriação social. Brasília: IBICT, UNE SCO, 2007. p. $185-212$.

Nonaka, I.; TAKeuchi, H. Criação de conhecimento na empresa: como as empresas japonesas geram a dinâmica da inovação. 11ª ed. Rio de Janeiro: Campus, 1997.

Organization for Economic Co-operation and Development. Survey on Knowledge M anagement Practioes for M inistries/ D epartments/ A gendes of C entral G overnment in OE CD M ember C ountries. Paris: OECD, 2002. D isponível em: <http:/ / www.oecd.org/ dataoecd/ 59/ 18/ 1946891.pdf>. Acesso em: 24 out. 2006.

Rod Rigues, S. B. D e fábricas a loja de conhecimento: as universidades e a desconstrução do conhecimento sem cliente. In: Fleury, M. T. L.; O liveira JR., M. M. (O rg.). G estão estratégica do conhecimento: integrando aprendizagem, conhecimento e competências. São Paulo: Atlas, 2001. p. 86-101.

São Paulo (Estado). Secretaria D e Estado Da Saúde - SP. Boletim do Instituto de Saúde, São Paulo: Instituto de Saúde, n. 30, p. 3-39, ago. 2003.

Stewart, T. A. A riqueza do conhecimento: 0 capital intelectual e a nova organização. Rio de Janeiro: Campus, 2002.

Sveiby, K. E. G estão do conhecimento - As lições dos pioneiros. [2001]. Disponível em: <http:/ / www.sveiby.com/ Portals/ 0/ articles/ Pioner.pdf>. Acesso em: 24 out. 2006.

Terra, J. C. C. Gestão do conhecimento: aspectos conceituais e estudo exploratório sobre práticas de empresas brasileiras. In: Fleury, M. T. L.; O liveira JR., M. M. (O rg.). $\mathrm{G}$ estão estratégica do onhecimento: integrando aprendizagem, conhecimento e competências. São Paulo: Atlas, 2001. p. 212-241.

Terra, J. C. C. G estão do Conhecimento: o grande desafio empresarial: uma abordagem baseada no aprendizado e na criatividade. São Paulo: Negócio, 2001. 


\section{Resumo - Resumen - Abstract}

\section{Gestão do conhecimento em organizações públicas de saúde no Brasil: diagnóstico de práticas \\ $\mathrm{G}$ erson Rosenberg, Pierre 0 hayon e Fábio Ferreira Batista}

As organizações públicas e privadas vivem diante de um cenário complexo, em que os fatores econômicos e sociais de alcance mundial são responsáveis pela sua reestruturação. Nesse contexto, a G estão do Conhecimento (GC) se torna um valioso instrumento estratégico para avida das pessoas edas organizações a que pertencem. A criação ea implantação de processos que gerem, armazenem, gerenciem e disseminem o conhecimento representam o mais novo desafio a ser enfrentado pelas organizações. 0 estudo realizado junto a Centros de Pesquisa Clínica (unidades vinculadas principalmente a Hospitais Universitánios) e organizações públicas de saúde integrantes do Programa Nacional de G estão Públicae D esburocratização (GESPÚBLICA) tem como objetivo verificar como o temaGC estásendo tratado. 0 artigo identifica as práticas de GC empregadas pelas organizações de saúde abrangidas pelo estudo e sugere recomendações para uma disseminação mais eficaz do Modelo de Excelência em G estão Pública.

Palavras-chave: gestão do conhecimento, administração pública, qualidade

\section{Gestión del conocimiento en organizaciones públicas de salud en Brasil: diagnóstico de conductas}

$\mathrm{G}$ erson R osenberg, Pierre 0 hayon y Fábio Ferreira Batista

Las organizaciones públicas y privadas enfrentan un complejo escenario dondefactores económicos y sociales, de alcance mundial, son directamente responsables por su reestructuración. D entro de este contexto, la G estión del Conocimiento (GC) se presenta como un valioso instrumento estratégico para la vida de las personas y de las organizaciones de las cuales hacen parte. La creación e implantación de procesos que generen, archiven, gerencien y diseminen el conocimiento, representan el más reciente desafío a ser enfrentado por las mismas. El estudio realizado junto a diferentes Centros de Investigación Clínica (unidades principalmentevinculadas a Hospitales Universitarios) y organizaciones públicas de salud que hacen parte del ProgramaNacional deG estión Públicay Desburocratización (GESPÚBLICA), tiene como objetivo, verificar como el tema GC está siendo tratado. El artículo identifica las prácticas de GC utilizadas por las organizaciones de salud abarcadas en el estudio y realiza recomendaciones para una diseminación más eficaz del Modelo de Excelencia en G estión Pública.

Palabras-clave: gestion del conocimiento , administración pública, calidad

\section{Knowledge management in Brazilian public health organizations: a diagnosis of practices}

G erson R osenberg, Pierre 0 hayon and Fábio Ferreira Batista

The public and private organizations are in complex scenario, where the economical and social glo bal factors are responsible for their restructuring. In this context, Knowledge Management (KM) becomes an important strategic instrument for the people's life and organizations to which they belong. The creation and implantation of processes which generate, store, manage and disseminate knowledge represent the most recent challenge for the organizations. The study carried out in Clinical Research Centers (units linked mainly to University Hospitals) and public health organizations, joined in the National Program of Public Administration in Brazil (G ESPÚBLICA), has as objective to verify how $\mathrm{KM}$ is being employed. In this article, $\mathrm{KM}$ practices used by the health organizations considered in the study are identified. Finally, recommendations are given for a more effective dissemination of the Model of Excellency for Public Administration.

Keywords: Knowledge management, public administration, quality 


\section{Gerson Rosenberg}

Engenheiro químico e tecnologista em saúde pública da Fundação O swaldo Cruz (Fiocruz/ Ministério da Saúde) com doutorado e mestrado pela Escola de Química da Universidade Federal do Rio de Janeiro e especialização em Gestão pela Qualidade Total pela Universidade Federal Fluminense (UFRJ). Cursando o pós-doutorado no Instituto de Economia da UFRJ.

Contato: gerson@fiocruz.br

Pierre O hayon

Graduado, mestre e doutor pela Faculdade de Economia e Administração da Universidade de São Paulo (FEA/ USP). Além de professor associado da FACC/ UFRJ, no D epartamento de Contabilidade, integra ainda o corpo docente do Programa de Pós-Graduação em Ciência da Informação do Instituto Brasileiro de Informação em Ciência e Tecnologia (Ibict), do Ministério da Ciência e Tecnologia (MCT). Pesquisador do CNPq e membro do International Committee on $\mathrm{N}$ on-Conventional Materials and Technologies.

Contato: pohayon@facc.ufrj.br

Fábio Ferreira Batista

Doutorando em Ciência da Informação pela Universidade de Brasília (UnB); Bacharel em Relações Internacionais $(\mathrm{UnB}) ;$ Mestre em História (UnB); Mestre em Relações Internacionais (School of Advanced International Studies da Johns Hopkins University - SAIS/ JHU); Especialista em Administração Pública (Fundação Getúlio Vargas FGV). Técnico de Planejamento e Pesquisa do Instituto de Pesquisa Econômica Aplicada (IPEA).

Contato: fabio.batista@ipea.gov.br 